\title{
A case of paraspinal pyomyositis in a healthy young man
}

\author{
Authors: Awanish Choudhary, ${ }^{\mathrm{A}}$ Jeyanthy Rajkanna, ${ }^{\mathrm{A}}$ Samson 0 Oyibo ${ }^{\mathrm{A}}$ and Sarah Choudhury ${ }^{\mathrm{A}}$
}

\section{Background}

Pyomyositis is a purulent infection of skeletal muscle that arises from haematogenous bacterial spread. It has always been thought to be an infection of the tropics, but has been recognised in temperate climates with increasing frequency. ${ }^{1}$ Pyomyositis has also been described among athletes performing vigorous exercise, suggesting the potential role of minor muscle damage in the pathogenesis of the disease. ${ }^{2,3}$ Men are more commonly affected than women. Predisposing factors for pyomyositis include immunodeficiency, trauma, intravenous drug use, concurrent infection, malnutrition, diabetes mellitus and malignancy. Diagnosis can be delayed if the affected muscle is deeply situated and local signs are not apparent. Initial serum inflammatory markers may be normal. We describe a case of pyomyositis in an unusual area in a healthy young man.

\section{Case presentation}

A 19-year-old man presented with severe back pain and fever. The pain was worse on movement. He had injured his left knee 10 days ago and sustained a minor abrasion while playing a contact sport. He had left knee swelling since then. He was a fit and well individual with no relevant past medical history. Examination revealed a swollen and tender left knee joint with a healing abrasion. Blood tests showed a raised C-reactive protein of $147 \mathrm{mg} / \mathrm{L}$, with normal full blood count and kidney function test. His HIV test was negative, blood glucose levels and creatinine phosphokinase levels were normal. An ultrasound of his kidneys demonstrated normal kidney anatomy but also revealed a post-micturating residual urine volume of $167 \mathrm{~mL}$ in the urinary bladder, suggesting urinary retention. Computed tomography of the abdomen and pelvis did not show any abnormality. Magnetic resonance imaging (MRI) of the spine demonstrated an extensive high-signal area in the right-sided paraspinal muscles (Fig 1). Subsequent blood cultures grew Staphylococcus aureus sensitive to flucloxacillin. Culture from the left knee aspirate was negative. Echocardiography ruled out endocarditis. He was treated with intravenous flucloxacillin as an inpatient. Follow-up MRI of the spine revealed a smaller area of infection more centred around the right facet joints of the third and fourth lumbar vertebral bones, consistent with resolving pyomyositis but residual lumbar facet joint septic

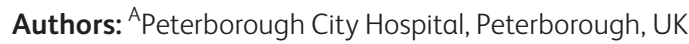

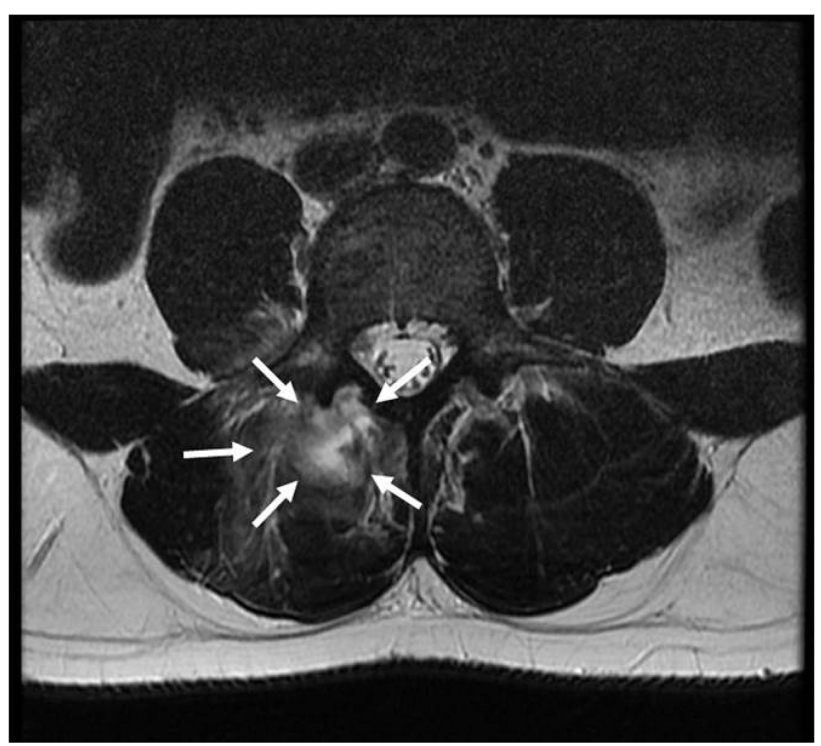

Fig 1. Magnetic resonance imaging of the lumbar spine showing an abscess in right paraspinal muscle.

arthritis. He was treated with intravenous ceftriaxone on an outpatient basis for 8 weeks thereafter.

\section{Conclusion}

Pyomyositis predominantly affects the muscles of the lower limbs, although it can also involve muscles of the upper limb, trunk and spine. Staphylococcus aureus is the commonest bacterial cause of pyomyositis, followed by group A streptococci infection. ${ }^{4} \mathrm{MRI}$ is the optimal imaging technique. It is highly sensitive for muscle inflammation and frank abscess, and can demonstrate the extent of tissue involvement. Image-guided percutaneous drainage is an option for many patients and can be useful both to secure a microbiological diagnosis and as a therapeutic measure when combined with antimicrobial therapy. The duration of antimicrobial therapy should be tailored around clinical and $\mathrm{r}$ adiographic improvement. ${ }^{5}$ Our patient was healthy and had no predisposing risk factors for the pyomyositis, which occurred distant from the site of initial trauma.

\section{Conflicts of interest}

None declared. 


\section{References}

1 Gibson RK, Rosenthal SJ, Lukert BP. Pyomyositis. Increasing recognition in temperate climates. Am J Med 1984;77:768-72.

2 Burkhart BG, Hamson KR. Pyomyositis in a 69-year-old tennis player. Am J Orthop (Belle Mead NJ) 2003;32:562-3.
3 Jayoussi R, Bialik V, Eyal A, Shehadeh N, Etzioni A. Pyomyositis caused by vigorous exercise in a boy. Acta Paediatr 1995;84:226-7.

4 Koutures CG, Savoia M, Pedowitz RA. Staphylococcusaureus thigh pyomyositis in a collegiate swimmer. Clin J Sport Med 2000;10:297-9.

5 Bickels J, Ben-Sira L, Kessler A, Wientroub S. Primary pyomyositis. J Bone Joint Surg Am 2002;84:2277-86. 